See discussions, stats, and author profiles for this publication at: https://www.researchgate.net/publication/335183560

\title{
GrassQ - A holistic precision grass measurement and analysis system to optimize pasture based livestock production
}

Conference Paper · January 2019

DOI: $10.13031 /$ aim. 201900769

CITATIONS

0

7 authors, including:

Darren J Murphy

Cork Institute of Technology

6 PUBLICATIONS 2 CITATIONS

SEE PROFILE

Michael D Murphy

Cork Institute of Technology

52 PUBLICATIONS 285 CITATIONS

SEE PROFILE

Some of the authors of this publication are also working on these related projects:
READS

36

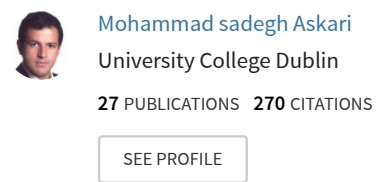

Project Predicting energy and water consumption on dairy farms through statistical analysis and machine-learning methods View project

Project $\quad$ GrassQ View project 


\title{
GrassQ - A holistic precision grass measurement and analysis system to optimize pasture based livestock production
}

\author{
Darren J Murphy 1,2, Bernadette O' Brien ${ }^{1}$, Mohammad Sadegh Askari ${ }^{3,4}$, Tim McCarthy ${ }^{3}$, Aidan \\ Magee $^{3}$, Rebekah Burke ${ }^{3}$, Michael D Murphy ${ }^{2}$ \\ ${ }^{1}$ Teagasc, Animal \& Grassland Research and Innovation Centre, Moorepark, Fermoy, Co. Cork, Ireland \\ ${ }^{2}$ Department of Process, Energy and Transport Engineering, Cork Institute of Technology, Cork, Ireland \\ ${ }^{3}$ Department of Computer Science, National University of Ireland Maynooth, Co. Kildare, Ireland \\ ${ }^{4}$ University of Zanjan, Zanjan, Iran \\ michaeld.murphy@cit.ie \\ Written for presentation at the \\ 2019 ASABE Annual International Meeting \\ Sponsored by ASABE \\ Boston, Massachusetts \\ July 7-10, 2019
}

ABSTRACT. GrassQ is a holistic grassland decision support system (DSS) that encapsulates a range of measurement technologies to provide yield and quality data to a cloud based platform, which can provide users with real time management information in the field. GrassQ aims to promote precision agricultural concepts within the pasture based livestock industry. Accurate measurement and allocation of fresh pasture to grazing herds on a daily basis is essential in increasing efficiency. Novel systems of measuring grass yield and quality were developed at the Moorepark Animal and Grassland Research Centre in Cork, Ireland, over the grass growing seasons of 2017 and 2018. Measurement systems included ground based and remote sensing techniques. The prototype GrassQ DSS was designed to process datasets uploaded from all proposed measurement systems. Measurement parameters were compressed sward height (CSH) (mm), herbage mass (HM) $(\mathrm{kgDM} / \mathrm{ha})$, dry matter $(D M)(\mathrm{g} / \mathrm{kg})$ and crude protein $(C P)(\mathrm{g} / \mathrm{kg})$. Ground based measurements were recorded using a smart rising plate meter (RPM) and lab based near infrared spectroscopy (NIRS). Multispectral remote sensing was carried out using an unmanned aerial vehicle (UAV), and data from the European Union's Sentinel-2 satellite (S2). Reference analyses for all prediction models were carried out at Moorpark's Grassland Laboratory and all sample locations were geotagged to enable spatial mapping of all parameters. The GrassQ prototype DSS is currently operational, including a number of preliminary grass quantity and quality prediction models. The complete Grass DSS will be is launched upon final validation.

Keywords. Decision Support System, Grassland Management, Precision Grazing, Rising Plate Meter, Remote Sensing, Near Infrared Spectroscopy

\footnotetext{
The authors are solely responsible for the content of this meeting presentation. The presentation does not necessarily reflect the official position of the American Society of Agricultural and Biological Engineers (ASABE), and its printing and distribution does not constitute an endorsement of views which may be expressed. Meeting presentations are not subject to the formal peer review process by ASABE editorial committees; therefore, they are not to be presented as refereed publications. Publish your paper in our journal after successfully completing the peer review process. See www.asabe.org/JournalSubmission for details. Citation of this work should state that it is from an ASABE meeting paper. EXAMPLE: Author's Last Name, Initials. 2019. Title of presentation. ASABE Paper No. ---. St. Joseph, MI.: ASABE. For information about securing permission to reprint or reproduce a meeting presentation, please contact ASABE at www.asabe.org/permissions (2950 Niles Road, St. Joseph, MI 49085-9659 USA). ${ }^{1}$
} 


\section{Introduction}

Pasture based farming has declined in recent decades in comparison to feed lot based systems (Huyghe et al., 2014), but in more recent years grass based meat and milk have seen a resurgence in consumer demand due to noted environmental and health benefits (Daley et al., 2010; Tilman and Clark, 2014). Grass based systems have the potential to be more economically sustainable but are hindered by reduced feed controllability, due to the spatial heterogeneity of grassland swards (Shalloo et al., 2005). Accurate measurement and allocation of fresh pasture to the grazing herd on a daily basis is essential in increasing the efficiency of grass based agricultural systems. Beukes et al. (2019) found that regular grass measurements can improve farm profits by up to $15 \%$ through increased feeding consistency, reduced feed imports and improved grassland management. Research in Ireland has indicated the value of increasing fresh grass utilization in a grazing herd’s diet to be in the range of $€ 160 /$ ha (Dillon, 2011) to $€ 267 /$ ha (French et al., 2015). There are a range of conventional grass yield measurement technologies currently on the market, but the limitations of such devices in terms of accuracy have been highlighted (O'Donovan et al., 2002; Sanderson et al., 2001). Furthermore, there is no definitive grass measurement sampling protocol that accounts for spatial heterogeneity, operator bias or labor cost. More recently an increasing amount of research has focused on airborne and ground based imagery and sensing methods for predicting grass yield and quality; such methods could cover large sampling areas with minimal labor requirements (Rueda-Ayala et al., 2019). Ancin-Murguzur et al. (2019) showed how hyperspectral imagery captured by satellite and ground based sensors can estimate grass yield with reasonable accuracy. Studies have indicated the potential application of remote sensing technologies for predicting and mapping grass quality parameters (Beeri et al., 2007; Knox et al., 2011). Further studies have evaluated the use of field and laboratory based NIRS sensors to rapidly analyze grass quality (Alomar et al., 2009; Pullanagari et al., 2011; Reddersen et al., 2013, 2014; Zeng and Chen, 2018). In addition to new measurement technologies, meteorological and seasonal data have been utilized to model grass growth and also improve the accuracy of conventional measurement systems, such as the rising plate meter (RPM) (Nakagami and Itano, 2014; Romera et al., 2010; Ruelle et al., 2018).

With the wide range of measurement technologies currently on the market and in research, there is growing scope for decision support systems that can process a large amount of empirical data from a range of sources, with the aim of streamlining data processing and aiding on-site decision making. PastureBase Ireland was recently launched as a cloud platform that stores and processes grass yield data to aid farmers in making both daily and seasonal grass management decisions (Hanrahan et al., 2017).

GrassQ is a European wide project funded by ICT-AGRI and ERA-NET that includes partners in Ireland, Denmark Finland and Switzerland ( $\mathrm{O}^{\prime}$ Brien et al., in press). The aim of this project is to develop a holistic precision grassland measurement and management system that encompasses both ground based and remote sensing measurement technologies. GrassQ will be structured upon a web based DSS designed to streamline data management and aid decision making for both industry and research. The DSS will be a cloud based data management and processing system capable of managing geospatial data from the range of measurement tools being researched as part of this project. Measurement research includes space borne and airborne multispectral sensing, laboratory based NIRS and ground based compressed sward height (CSH). Data captured from local metrological stations will further be utilized by GrassQ to enhance modeling precision. Outputs from GrassQ will include high quality, real time, geo-referenced information on pasture yield and quality that can be used to aid grassland management decision making. The main goals in developing this system are to optimize grass quality, utilization and profitability while simultaneously minimizing labor requirements and subjectivity in decision making.

\section{Material and Methods}

\section{GrassQ Decsision Support System Overview}

The basic structure of the GrassQ DSS consists of four layers including the sensor, cloud storage, geoinformatics and decision support layer, which are outlined in the infographic in Figure 1. The sensor layer includes all measurement technologies incorporated in GrassQ and all data acquired from this layer is uploaded and stored on the cloud platform. All grass quality and quantity prediction models developed as part of GrassQ are integrated within the geoinformatics layer, where geo-tagged information outputs such as user friendly result forms, maps, and alerts can be generated in real time. The decision support layer allows data outputs from the geoinformatics layer to be visualized via a user friendly interface either through the GrassQ web browser portal or via the GrassQ smartphone application. 


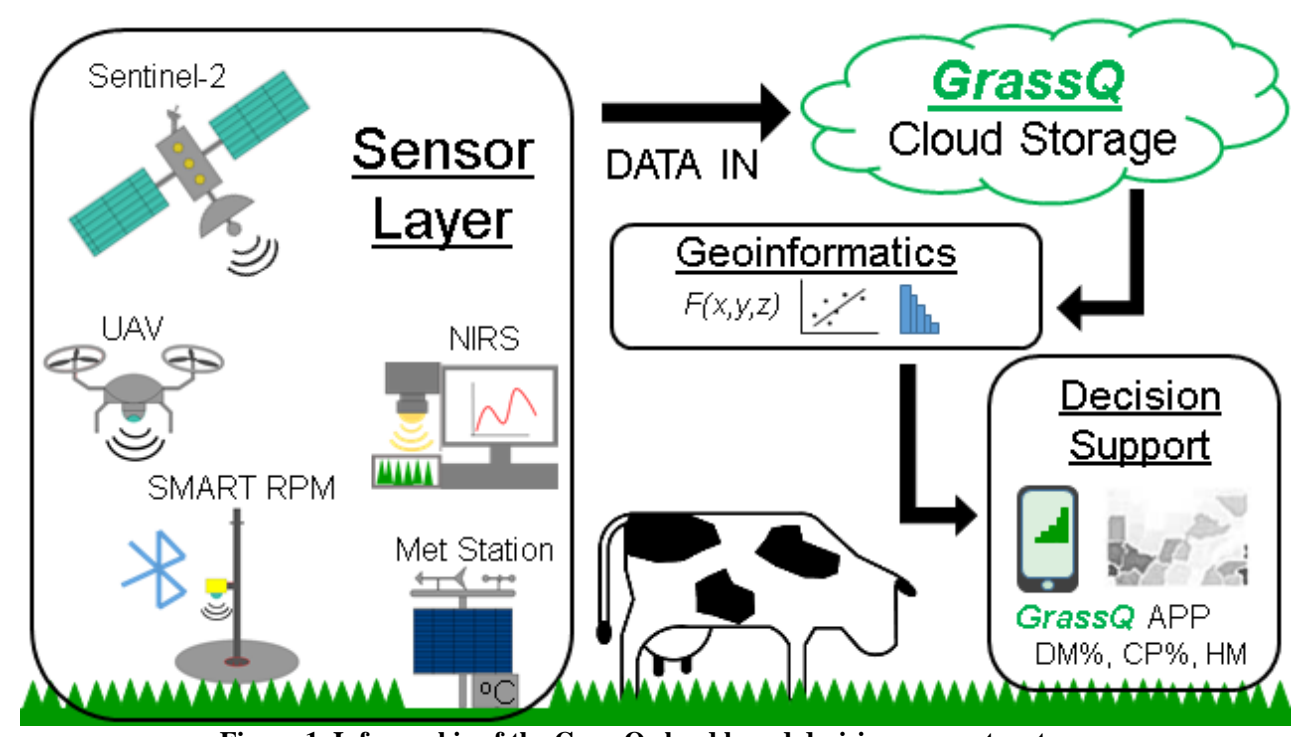

Figure 1. Infographic of the GrassQ cloud based decision support system

\section{Ground Based Data}

Trial work commenced on developing the measurement systems included in the sensor layer in March 2017. Ground based reference measurements were taken on controlled trial plots $\left(7.5 \mathrm{~m}^{2}\right)$ and grazed paddocks ( $\left.1 \mathrm{ha}\right)$ at the Teagasc Animal and Grassland Research Institute at Moorepark, Fermoy, Co. Cork Ireland (50 $\left.7^{\prime} \mathrm{N}, 18^{\circ} 16^{\prime} \mathrm{W}\right)$. Measurement parameters included compressed sward height (CSH), dry mater (DM), herbage mass (HM) and crude protein (CP). The Grasshopper (True North Technologies Ltd, Shannon, Ireland), an automated RPM controlled by a smart phone application (Grasshopper 1.2) with GPS capabilities was used to record CSH. Data was acquired on a weekly basis during the grazing seasons of 2017 and 2018 as outlined in Murphy et al. (2018). Blanket height samples were taken on plots $(\mathrm{n}=39)$ and paddocks $(\mathrm{n}=$ 320 ) to estimate true mean CSH and the spatial heterogeneity of CSH and HM. Laboratory based Spectral analysis recorded at $2 \mathrm{~nm}$ intervals in the range of $1100 \mathrm{~nm}-2500 \mathrm{~nm}$ was performed on all fresh herbage samples using a FOSS 6500 spectrometer (FOSS-NIR System DK, HillerØid, Denmark) as outlined in Murphy et al. (in press). Wet chemistry reference analysis was then performed to determine DM and HM by oven drying as in McEvoy et al. (2011). Reference data for CP was obtained by Leco analysis (Leco FP-528; Leco Corporation, St Joseph, MI, USA). Metrological data for each measurement date was recorded by the on-site Met Éireann synoptic weather station at Moorepark.

\section{Optimum Sampling Protocol}

To investigate potential optimum ground based sampling rates and patterns, a retrospective sampling reduction algorithm was developed using Visual Basics Applied on Microsoft Excel (2013). The algorithm estimated CSH prediction error based upon simulated reduced sampling rates. Sampling simulations were carried out in a random stratified manner as recommended by Webster and Lark (2012). An exponential relationship between sampling rate and average CSH prediction error was then developed enabling economic analysis of a number of potential optimum sampling rates to be carried out.

\section{Remote Sensing}

Remote sensing measurements were performed on both plots and paddocks on five separate dates coinciding with ground based measurements over the two year trial work period at Moorepark. Data was captured by two separate methods on each sampling date. Firstly, data was acquired using the multispectral sensor instrument on board S2; this instrument captures high resolution imagery $(10 \mathrm{~m}, 20 \mathrm{~m}, 60 \mathrm{~m})$ in the visible near infrared (VNIR) and short wave infrared regions (SWIR) $(440 \mathrm{~nm}-2200 \mathrm{~nm})$ of the electromagnetic spectrum (ESA, 2019). Secondly, airborne spectral and RGB imaging was carried out using a Sequoia Multispectral sensor (Parrot Drones SAS, Paris, France) fixed to a UAV, which captured spectral data at four bands; green $(550 \mathrm{~nm})$, red $(660 \mathrm{~nm})$, red edge $(735 \mathrm{~nm})$ and near infrared (790 nm) (Parrot, 2019).

\section{Modeling}

Partial least squares regression (PLSR) was employed to model the relationship between CSH and HM, both on an annual and seasonal basis using Microsoft Excel (2013). Multiple Linear regression (MLR) was employed to investigate if the addition of metrological data could further improve the prediction accuracy of the relationship between CSH and HM. NIRS spectra from laboratory analysis were modeled against wet chemistry reference data by means of modified partial least squares regression using WinISI 4 chemometric software (ISI, Port Matilda, Pennsylvania, USA). Data from UAV surveys was transformed into surface reflectance images and S2 datasets were downloaded in the form of atmospherically corrected 'Level 2A products'. Geometric and radiometric corrections were applied to multispectral data as pre-processing procedures prior to PLSR modeling using Unsrambler software (version X10.4.1; CAMO software, Woodbridge, NJ, USA). The preprocessed spectral data was further modeled using MLR on SPSS v. 21 (SPSS Inc.). Initially a range of 20 spectral indices were accessed for CP and DM prediction performance. The best performing indices were then selected for further model 
development.

\section{GrassQ Cloud Platform}

A cloud based DSS platform was developed using Amazon Web Services (Amazon Web Services, Inc.) to store and process datasets uploaded from all measurement tools included in this research and to disseminate this data in an intuitive and user friendly manner. Furthermore the DSS encapsulates geospatial data uploaded from reference surveys taken at farm level along with open source boundary, elevation, geological and environmental data. The platform was designed to be accessible both as a web portal and as a user friendly smart phone application, to enable access to real time data and decision support in the field. There are two tiers of access; one for farmers that provides pre-processed data in an intuitive manner to streamline decision making, the other for researchers that enables raw datasets to be downloaded for further processing and modeling.

\section{Results and Discussion}

The GrassQ project entered its final year of testing in 2019 and all findings presented in the following sections are preliminary.

\section{Rising Plate Meter}

The preliminary PLSR relationships between CSH and HM for data collected over the 2017 and 2018 grazing seasons are illustrated in Figure 2. The pooled dataset $(\mathrm{n}=1074)$ was sub-divided into three seasonal datasets to investigate seasonal variations in the relationship between CSH and HM. This dataset was divided into the following seasons; spring (April to June, $n=478$ ), summer (July - September, $n=380$ ) and winter (October - March, $n=216$ ). In Figure 2 it is clear that there is a considerable change in the relationships and coefficients of determination between the spring $\left(\mathrm{R}^{2}=0.816\right)$ and summer regressions $\left(R^{2}=0.441\right)$. These findings are most likely as a result of the noted change in perennial ryegrass sward morphology and structure as the plant enters the inflorescence growth stage from mid to late summer. These findings are in agreement with a number of other studies (Defrance et al., 2004; Ferraro, 2012; Nakagami and Itano, 2014). The relationships presented in Figure 2, will be extended and validated over the 2019 grazing season and will be used to benchmark an MLR incorporating both seasonal and meteorological data. All models will be validated against each other to select the most suitable to be incorporated as part of the GrassQ DSS.
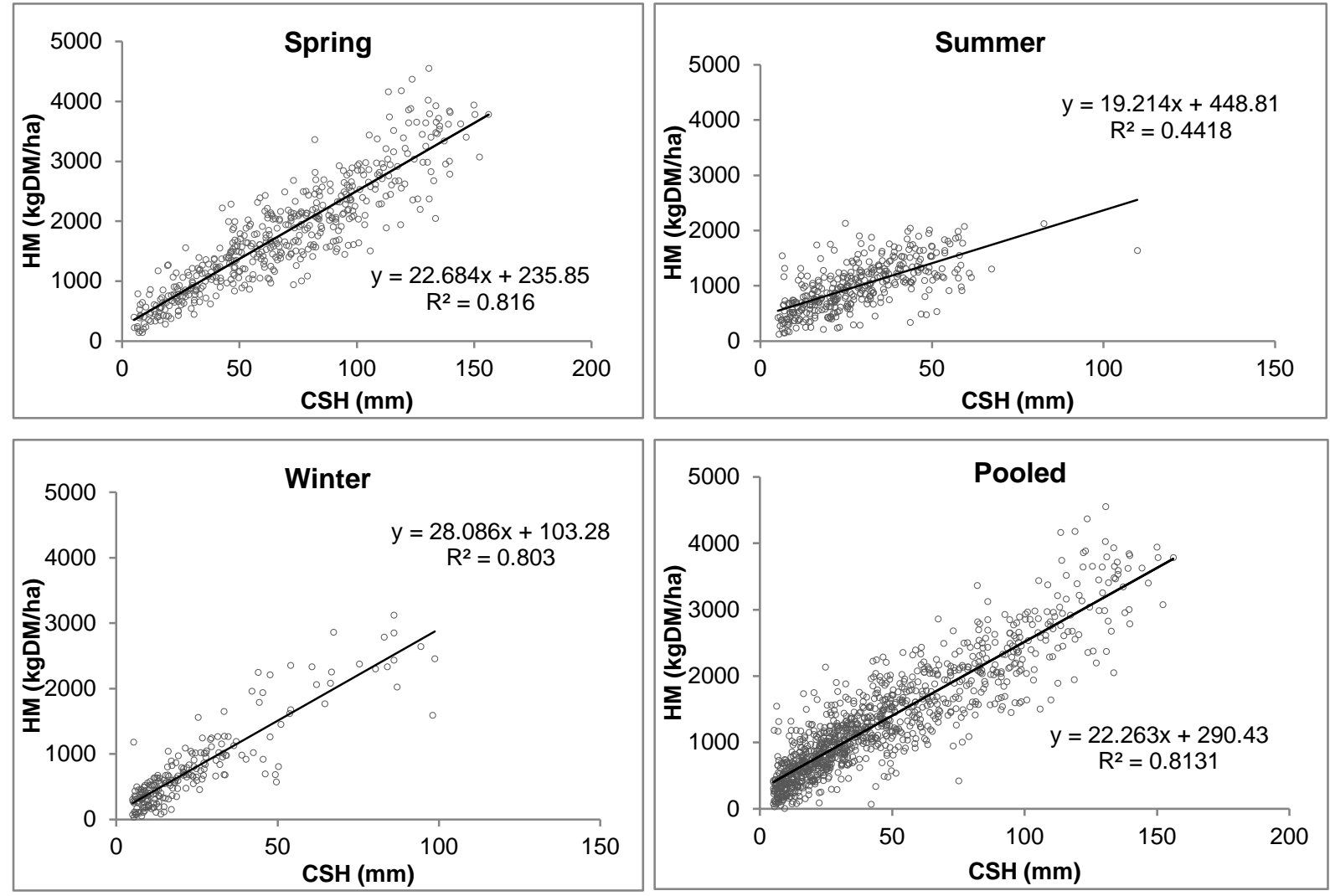

Figure 2. GrassQ linear seasonal and pooled herbage mass prediction models

NIRS

An NIRS calibration for the prediction of CP and DM to determine the quality of fresh grass in near real time as part of GrassQ is in the final stages of development. Results of a preliminary equation were presented in Murphy et al. (in press). 
The preliminary findings indicate that fresh grass quality can be predicted with acceptable accuracy $\left(\mathrm{R}^{2}=0.93, \mathrm{R}^{2}=0.89\right.$ for DM and CP respectively) within a time frame of three to five minutes, in comparison to wet chemistry analysis. The GrassQ DSS is being developed to incorporate NIRS data in consideration of the increasing level of research that is being applied to the area of online portal NIRS sensors that can rapidly evaluate herbage quality in the field (dos Santos et al., 2013; Reddersen et al., 2013). It is envisaged that the fresh grass NIRS model developed as part of this study will be used to benchmark future online NIRS technologies as part of GrassQ.

\section{Sampling Labor Utilization Tool}

Preliminary findings from a study to investigate an optimum sampling protocol for the RPM as part of the GrassQ project were presented in Murphy et al. (2018). An optimum targeted level of sampling accuracy was found to be in the region between $5-10 \%$ SE. Sampling to this level of accuracy required a theoretical sampling rate between $189-512$ samples/ha; this would optimize both sampling cost and accuracy. Based on these findings and further work carried out during the 2018 grazing season on grazed paddocks, a labor utilization tool is being developed to be incorporated into the GrassQ system. The tool will utilize uploaded sward and GPS data to generate optimal sampling protocols for selected pastures via an interactive map. Generated protocols will include optimal sampling routes and rates dependent upon a range of sward factors and the desired levels of sampling accuracy and cost.

\section{Remote Sensing}

The initial phase of remote sensing modeling has been completed with results indicating that HM can be estimated from S2 $\left(R^{2}>0.7\right)$ and UAV $\left(R^{2}>0.8\right)$ multispectral data sets using stepwise MLR. Yield maps for S2 and UAV data sets generated from the prototype GrassQ DSS are illustrated in Figure 3 and Figure 4, where variations in HM density are clearly distinguishable between different treatment groups and pastures. A number of multispectral bands were identified as significant for predicting HM and selected for further research; these included red edge, NIR and green wavelength bands. With regard to predicting HM from multispectral UAV data, modified chlorophyll (MCAR), modified triangle vegetation index and modified non-linear index (MNLI) were selected as the top performing indices and selected for further investigation. The most significant bands in terms of modeling HM from S2 data were band 11 (centered at $1610 \mathrm{~nm}$ ) and band 12 (centered at $2190 \mathrm{~nm}$ ). Grass quality prediction models for predicting CP are still in the development stage although initial results indicate acceptable accuracy $\left(\mathrm{R}^{2}>0.8\right)$.
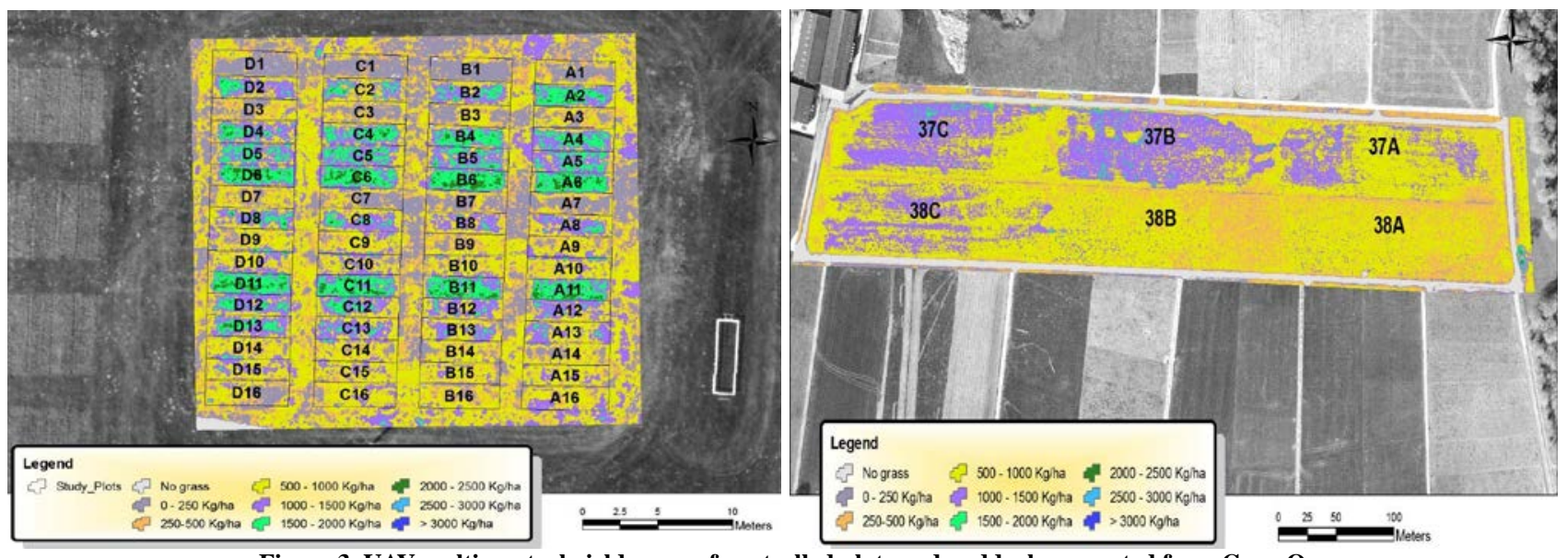

Figure 3. UAV multispectral yield maps of controlled plots and paddock generated from GrassQ 


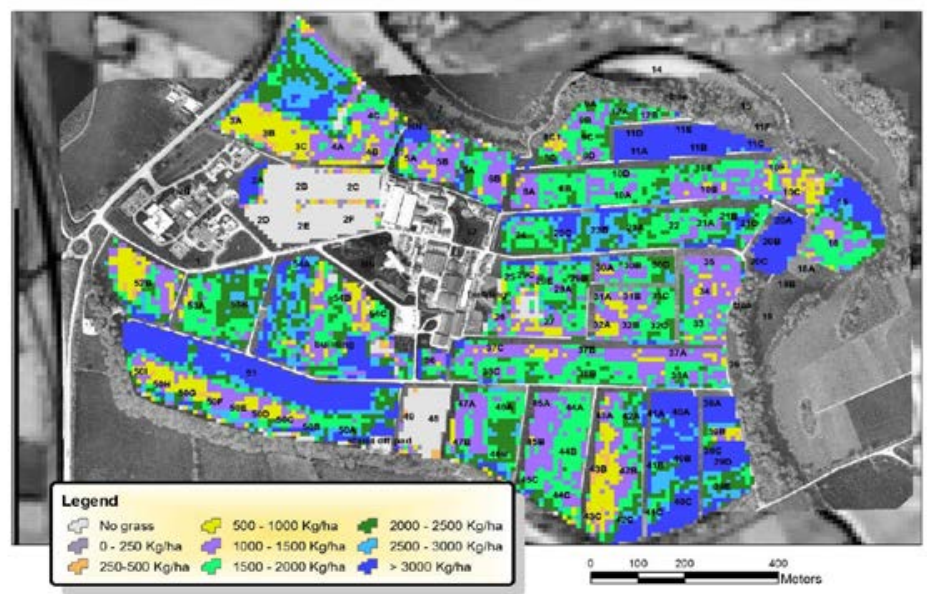

Figure 4. Sentinel-2 multispectral yield maps of Moorepark paddock generated from GrassQ

\section{GrassQ Cloud Base Decision Support System}

The GrassQ web based DSS prototype was launched in October 2018 and an image of the user interface can be seen in Figure 5. The website can be accessed at http://www.grassq.com and users must register an account before access is granted. The online GIS based interface enables users to pan through their grazing platform and select paddocks of interest. Users can create maps of desired features to visualize uploaded data processed by the systems geo-informatics layer. Maps and graphs of recorded surveys can also be downloaded. The online platform has two user interfaces to choose from, a more simplistic interface for basic grazing decision support and a research interface where raw data sets can be downloaded. The GrassQ smart phone application consists of a condensed version of the online platform and a prototype was launched in 2018. The GrassQ DSS is in the final stages of development and is due to be launched once final validation is completed. A preliminary evaluation of the GrassQ DSS prototype by farmers in 2018 outlined by Kaivosoja et al. (in press) received positive results, with farmers recognizing the benefits of the systems applications for grassland management support.

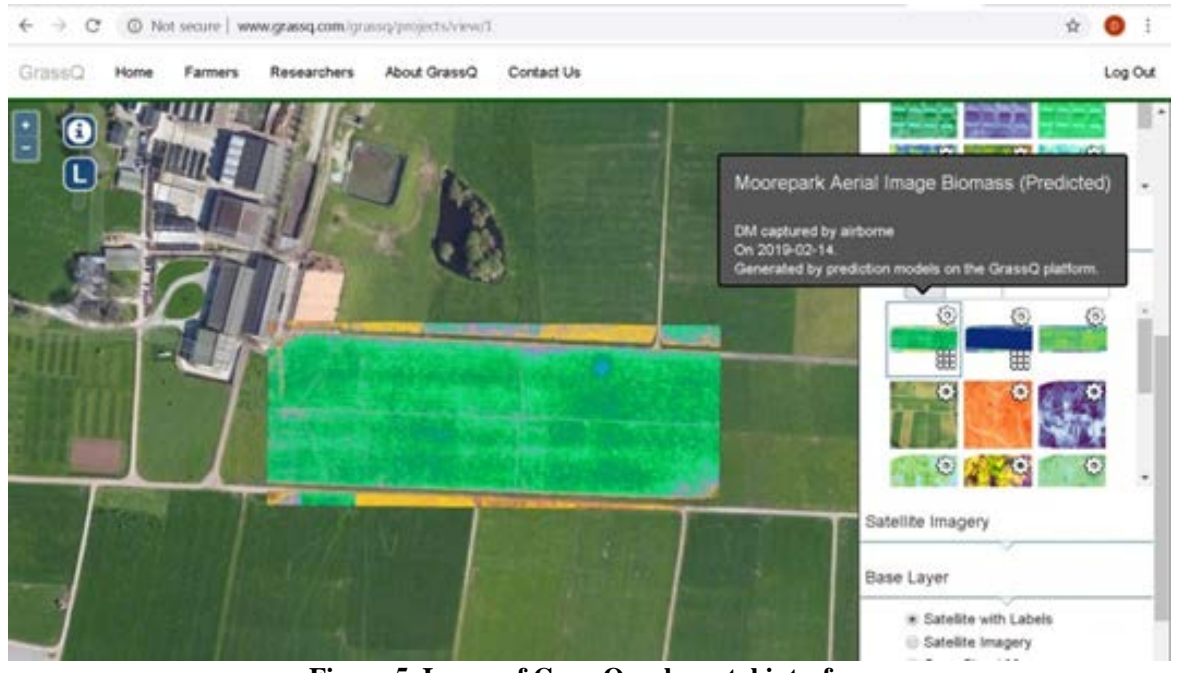

Figure 5. Image of GrassQ web portal interface

\section{Conclusion}

The prototype GrassQ holistic grassland DSS has been developed and is capable of storing and processing both ground based and remote sensing measurement data. Multiple grass quantity and quality prediction models for each measurement system incorporated in the GrassQ project have been prototyped and have achieved satisfactory thresholds of accuracy for use in grass based systems. Furthermore, a ground based grass measurement labor utilization tool that will be built into the GrassQ DSS, is under development to optimize sampling accuracy and cost. The GrassQ DSS and all included prediction models will be further developed and validated in the near future, along with the launch of the completed GrassQ smartphone application for dissemination of management information in the field. It is envisaged that GrassQ will promote precision agricultural concepts and increase efficiency within the pasture based livestock industry. 


\section{References}

Alomar, D., Fuchslocher, R., Cuevas, J., Mardones, R., \& Cuevas, E. (2009). Prediction of the composition of fresh grass pastures by near infrared reflectance or interactance-reflectance spectroscopy: Chilean Jouranl of Agricultural Research, 69(2), 198-206

Ancin-Murguzur, F. J., Taff, G., Davids, C., Tømmervik, H., Mølmann, J., \& Jørgensen, M. (2019). Yield estimates by a two-step approach using hyperspectral methods in grasslands at high atitudes: Remote Sensing, 11(4), 400

Beeri, O., Phillips, R., Hendrickson, J., Frank, A. B., \& Kronberg, S. (2007). Estimating forage quantity and quality using aerial hyperspectral imagery for northern mixed-grass prairie: Remote Sensing of Environment, 110(2), 216-225. https://doi.org/10.1016/j.rse.2007.02.027

Beukes, P. C., McCarthy, S., Wims, C. M., Gregorini, P., \& Romera, A. J. (2019). Regular estimates of herbage mass can improve profitability of pasture-based dairy systems: Animal Production Science, 59(2), 359-367. https://doi.org/10.1071/AN17166

Daley, C. A., Abbott, A., Doyle, P. S., Nader, G. A., \& Larson, S. (2010). A review of fatty acid profiles and antioxidant content in grass-fed and grain-fed beef: Nutrition journal, 9, 10-10. 10.1186/1475-2891-9-10

Defrance, P., Delaby, L., \& Seuret, J. M. (2004). Mieux connaître la densité de l'herbe pour calculer la croissance, la biomasse d'une parcelle et le stock d'herbe disponible d'une exploitation. Proc. Rencontres autour des Recherches sur les Ruminants 11 pp. 291-294. Paris

Dillon, P. (2011). The Irish dairy industry-Planning for 2020. Proc. National Dairy Conference 2011 'The Irish Dairy Industry: To 2015 and Beyond' pp. 1-24. Rochestown Park, Cork, reland

dos Santos, C. A. T., Lopo, M., Páscoa, R. N. M. J., \& Lopes, J. A. (2013). A Review on the Applications of Portable NearInfrared Spectrometers: Applied Spectroscopy, 67(11), 1215-1233. https://doi.org/10.1366/13-07228

ESA. (2019). MultiSpectral Instrument (MSI) Overview. Retrieved 12/04/2019, 2019, from https://sentinel.esa.int/web/sentinel/technical-guides/sentinel-2-msi/msi-instrument

Ferraro, F. P. (2012). Seasonal Variation in the Rising Plate Meter Calibration for Forage Mass: Agronomy Journal, 104(1), 1-6. 10.2134/agronj2011.0190

French, P., Hanrahan, L., \& Shalloo, L. (2015). Principles of sustainable dairy expansion. Proc. Moorepark '15 Irish Dairying - Sustainable Expanasion pp. 9-14.

Hanrahan, L., Geoghegan, A., O'Donovan, M., Griffith, V., Ruelle, E., Wallace, M., \& Shalloo, L. (2017). PastureBase Ireland: A grassland decision support system and national database: Computers and Electronics in Agriculture, 136, 193-201. http://doi.org/10.1016/j.compag.2017.01.029

Huyghe, C., De Vliegher, A., Van Gils, B., \& Peeters, A. (2014). Executive summary. In Grasslands and herbivore production in Europe and effects of common policies (pp. 7-13). Versailles Cedex, France: Editions Quae.

Kaivosoja, J., Niemeläinen, O., Näsi, R., Burke, R., McCarthy, T., O’Brien, B., . . Honkavaara, E. (in press). Evaluation of remote sensing technologies and cloud services to support grassland management. Proc. European Conferance on Precsission Livestock Farming University College Cork

Knox, N. M., Skidmore, A. K., Prins, H. H. T., Asner, G. P., van der Werff, H. M. A., de Boer, W. F., .. Grant, R. C. (2011). Dry season mapping of savanna forage quality, using the hyperspectral Carnegie Airborne Observatory sensor: Remote Sensing of Environment, 115(6), 1478-1488. https://doi.org/10.1016/j.rse.2011.02.007

McEvoy, M., O’Donovan, M., \& Shalloo, L. (2011). Development and application of an economic ranking index for perennial ryegrass cultivars: Journal of Dairy Science, 94(3), 1627-1639. http://doi.org/10.3168/jds.2010-3322

Murphy, D. J., O' Brien, B., \& Murphy, M. D. (2018). Development of a Labour Utilisation Decision Support Tool to Efficiently Measure Grass Herbage Mass Using a Rising Plate Meter. Proc. 2018 ASABE Annual International Meeting pp. 2-8. Detroit, MI:https://doi.org/10.13031/aim.201800806

Murphy, D. J., O' Brien, B., O’ Donovan, M., Condon, T., Claffey, A., \& Murphy, M. D. (in press). A preliminary near infrared spectroscopy calibration for the prediction of un-dried fresh grass quality. Proc. European Conference on Precision Livestock Farming. University College Cork

Nakagami, K., \& Itano, S. (2014). Improving pooled calibration of a rising-plate meter for estimating herbage mass over a season in cool-season grass pasture: Grass and Forage Science, 69(4), 717-723. 10.1111/gfs.12070

O' Brien, B., Murphy, D. J., Askari, M. S., Burke, R., Umstätter, C., Hart, L., . . McCarthy, T. (in press). Modelling precision grass measurements for a web-based decision platform to aid grassland management. Proc. European Conference on Precision Livestock Farming. University College Cork

O'Donovan, M., Dillon, P., Rath, M., \& Stakelum, G. (2002). A Comparison of Four Methods of Herbage Mass Estimation: Irish Journal of Agricultural and Food Research, 41(1), 17-27

Parrot, D. S. (2019). PARROT SEQUOIA. Retrieved 12/04/2019, 2019, from https://www.parrot.com/business-solutionsus/parrot-professional/parrot-sequoia\#parrot-sequoia-

Pullanagari, R., Yule, I., Tuohy, M., Dynes, R., \& King, W. (2011). Pasture quality measurement tools for decision making: Occasional Report, 24

Reddersen, B., Fricke, T., \& Wachendorf, M. (2013). Effects of sample preparation and measurement standardization on the 
NIRS calibration quality of nitrogen, ash and NDFom content in extensive experimental grassland biomass: Animal Feed Science and Technology, 183(3), 77-85. https://doi.org/10.1016/j.anifeedsci.2013.04.016

Reddersen, B., Fricke, T., \& Wachendorf, M. (2014). A multi-sensor approach for predicting biomass of extensively managed grassland: Computers and Electronics in Agriculture, 109, 247-260

Romera, A. J., Beukes, P., Clark, C., Clark, D., Levy, H., \& Tait, A. (2010). Use of a pasture growth model to estimate herbage mass at a paddock scale and assist management on dairy farms: Computers and Electronics in Agriculture, 74(1), 66-72. https://doi.org/10.1016/j.compag.2010.06.006

Rueda-Ayala, V., Peña-Barragán, J. M., Höglind, M., Bengochea-Guevara, J., \& Andújar, D. (2019). Comparing UAV-Based Technologies and RGB-D Reconstruction Methods for Plant Height and Biomass Monitoring on Grass Ley (Vol. 19).

Ruelle, E., Hennessy, D., \& Delaby, L. (2018). Development of the Moorepark St Gilles grass growth model (MoSt GG model): A predictive model for grass growth for pasture based systems: European Journal of Agronomy, 99, 80-91. https://doi.org/10.1016/j.eja.2018.06.010

Sanderson, M. A., Rotz, C. A., Fultz, S. W., \& Rayburn, E. B. (2001). Estimating forage mass with a commercial capacitance meter, rising plate meter, and pasture ruler: Agronomy Journal, 93(6), 1281-1286

Shalloo, L., Dillon, P., \& Murphy, J. J. (2005). A comparison of three systems of milk production with different land use strategies. InJ. J. Murphy (Ed.),Proc. Satellite Workshop of the XXth International Grassland Congress pp. 236. Cork, Ireland:http://dx.doi.org/10.3920/978-90-8686-554-3

Tilman, D., \& Clark, M. (2014). Global diets link environmental sustainability and human health: Nature, 515, 518. 10.1038/nature13959

Webster, R., \& Lark, M. (2012). Efficiency, economy and logistics. In Field sampling for environmental science and management (pp. 56). Milton Park, Abingdon, Oxon, UK: Routledge.

Zeng, L., \& Chen, C. (2018). Using remote sensing to estimate forage biomass and nutrient contents at different growth stages: Biomass and Bioenergy, 115, 74-81 